contrast, single-handed practices have been able to benefit from the Small Business Rate Relief Scheme, including a $£ 10,000$ grant, and this has greatly helped them to get through this crisis.

This contrast in fortunes begs one question: is it time for the profession to reconsider its fondness for larger practices and to cherish anew, its rich heritage of small, family practices?

C. Marks, Southampton, UK https://doi.org/10.1038/s41415-020-1930-y

\section{Coping well}

Sir, we read Drs Mijiti and Huojia's letter (Psychosomatic problems BDJ 2020; 228: 738 ) with deep empathy. Japan is gradually moving out of the emergency state but remains vigilant against the second wave of COVID-19. As the authors described, patients with chronic pain like Burning Mouth Syndrome (BMS) are at high risk for depression and anxiety with social isolation a possible risk for developing further psychosomatic symptoms. ${ }^{1}$

As of July 2020, two weeks after the re-opening of Psychosomatic Dentistry Clinic (Dental Hospital, Tokyo Medical and Dental University), we do not observe a growing number of patients with new oral psychosomatic disorders. Also, the pandemic chaos did not necessarily worsen our patients' condition. In contrast, some even told us that they felt better by being released from the stress of commuting and mental fatigue of workplace interpersonal relationships. We suppose here that BMS symptoms might not be simply connected with anxiety and depression, as do some psychiatric conditions. In most cases our BMS patients are coping well with this unusual situation, beyond our expectations.

The activities of our psychosomatic dental services were interrupted and reduced for several months, being considered as non-urgent. Almost all patients had to be followed up with telemedicine, including brief psychological counselling via telephone; continuing the prescriptions (mainly for antidepressants) via fax services, in cooperation with nearby pharmacies. Many patients expressed thanks for keeping up the medication while being able to avoid infection risks such that we suggest online consulting was useful for BMS patients during social distancing. It would also be useful in the event of a second wave of COVID-19 and other natural disasters, like earthquakes or typhoons.

Treating a dental patient with

psychosomatic problems is always difficult. ${ }^{2}$ However, given the many fundamental changes to daily lives in the current pandemic, we suggest expanding telehealth and telemedicine to follow up oral

psychosomatic patients at home. This also aligns with Dr Mijiti's idea of training dental hygienists to apply some psychological techniques of Cognitive Behavioural Therapy (CBT). ${ }^{3}$

\section{T. T. H. Tu, A. Toyofuku, Tokyo, Japan, H. Matsuoka, Hokkaido, Japan}

\section{References}

1. Piraccini $E$, Byrne $H$, Taddei $S$. Chronic pain management in COVID-19 Era. J Clin Anesth 2020; 65: 109852.

2. Toyofuku A. Psychosomatic problems in dentistry. Biopsychosoc Med 2016; 10: 14.

3. Matsuoka H, Chiba I, Sakano Y, Toyofuku A, Abiko Y. Cognitive behavioral therapy for psychosomatic problems in dental settings. Biopsychosoc Med 2017; 11: 18.

https://doi.org/10.1038/s41415-020-1931-x

\section{Temporary filling anaphylaxis}

Sir, a fit and well 33-year-old female presented to A\&E with signs and symptoms in keeping with anaphylaxis. Prior to this manifestation she had placed an 'at home' temporary filling into tooth 35 ; at examination she stated an allergy to cloves and thus we suspected that the material contained eugenol.

After stabilisation in A\&E, she was reviewed by our team. On discussion she decided against removal of the filling under rubber dam or the tooth itself. However, she re-presented approximately 48 hours later with recurrent symptoms of anaphylaxis at which point the tooth was extracted in a theatre setting under local anaesthetic; she made a full recovery.

We would like to highlight this concern due to the increased use of 'at home' filling kits by our patients, as a result of remote triage during the coronavirus pandemic. Here it is important to inform patients to check temporary filling material ingredients and update allergy status when advising such management.

G. Zakary, R. Major, A. Bhaiyat, York, UK https://doi.org/10.1038/s41415-020-1932-9

\section{Judgement and virtual reality}

Sir, dental education is changing as a result of the COVID-19 pandemic and we highlight two aspects of this to affect us as final year students.

We have been informed that dental foundation training (DFT) interviews will no longer be taking place and the recruitment process will be solely placed upon the situational judgement test. This consists of a selection of multiple-choice and ranking-based questions to represent challenging scenarios which dentists may encounter. The news has heightened anxiety levels in many students. As this is the only exam to decide undergraduate ranking among their peers, the amount of pressure placed upon them has been amplified.

Secondly, students in their later years of training are anxious as the lack of clinical exposure inevitably decreases confidence levels. We are fortunate to have experienced hapTEL (haptics in technology-enhanced learning). This award-winning virtual reality technology consists of glasses, a foot pedal and a drill giving students a realistic 3D experience with sound, touch and vision. Benefits include improvements in the positioning of the clinician, enhanced manual dexterity and early adaption to a clinical setting. Precision can be practised as the different layers of the tooth surface can be differentiated, improving tactile sensation. It also serves as a great tool to gain constructive feedback from tutors.

We believe this device would be beneficial to use as an adjunct to fill the gap of lost clinical time and that it should be implemented across other universities to enhance clinical practice to achieve optimum care for our patients.

S. Thakkar, S. Kadia, London, UK https://doi.org/10.1038/s41415-020-1933-8

\section{An opportunity}

Sir, with the increased success of identifying symptomatic COVID-19 cases followed by self-isolation, the main mode of transmission is going to be increasingly through asymptomatic spread. ${ }^{1}$ We are not routinely testing patients who have been selected for an aerosol generating procedure (AGP) and this is based (in Scotland) on the low prevalence of 